\title{
Two Promoter Rearrangements in a Drug Efflux Transporter Gene Are Responsible for the Appearance and Spread of Multidrug Resistance Phenotype MDR2 in Botrytis cinerea Isolates in French and German Vineyards
}

\author{
D. Mernke, S. Dahm, A.-S. Walker, A. Lalève, S. Fillinger, M. Leroch, and M. Hahn
}

First, second, sixth, and seventh authors: University of Kaiserslautern, Department of Biology, P.O. Box 3049, 67653 Kaiserslautern, Germany; and third, fourth, and fifth authors: INRA, UR 1290 BIOGER-CPP, Avenue Lucien Bretignières, BP 01, F78850 ThivervalGrignon, France.

Accepted for publication 3 May 2011.

\begin{abstract}
Mernke, D., Dahm, S., Walker, A.-S., Lalève, A., Fillinger, S., Leroch, M., and Hahn, M. 2011. Two promoter rearrangements in a drug efflux transporter gene are responsible for the appearance and spread of multidrug resistance phenotype MDR2 in Botrytis cinerea isolates in French and German vineyards. Phytopathology 101:1176-1183.

In French and German vineyards, Botrytis cinerea isolates with multiple fungicide resistance phenotypes have been observed with increasing frequencies. Multidrug resistance (MDR) results from mutations that lead to constitutive overexpression of genes encoding drug efflux transporters. In MDR2 and MDR3 strains, overexpression of the major facilitator superfamiliy transporter gene $m f s M 2$ has been found to result

from a rearrangement in the $m f s M 2$ promoter (type A), caused by insertion of a retroelement (RE)-derived sequence. Here, we report the discovery of another, similar RE-induced rearrangement of the $m f_{s} M 2$ promoter (type B) in a subpopulation of French MDR2 isolates. MDR2 isolates harboring either type A or type B mutations in $m f s M 2$ show the same resistance phenotypes and similar levels of $m f s M 2$ overexpression. RE sequences similar to those in $m f s M 2$ were found in low copy numbers in other but not all $B$. cinerea strains analyzed, including non-MDR2 strains. Population genetic analyses support the hypothesis that the two rearrangement mutations have only occurred once, and are responsible for the appearance and subsequent spread of all known MDR2 and MDR3 strains in French and German wine-growing regions.
\end{abstract}

Botrytis cinerea causes gray mold rot on more than 200 plant species (26). Under humid conditions, the fungus can cause serious yield and quality losses in fruit and vegetable production, including tomato, strawberry, and grape. To control gray mold in vineyards, regular fungicide treatments are common. Various fungicides with different modes of action are effective against $B$. cinerea. However, due to its great genetic diversity and its enormous capacity for asexual reproduction by means of conidiospores, B. cinerea has been classified as a "high-risk pathogen" that shows rapid development of fungicide resistance $(8,25)$.

In plant-pathogenic fungi, including $B$. cinerea, the most important mechanism of resistance against site-specific fungicides is the alteration of the target protein by point mutations. If these mutations do not impair the function of the target, highly resistant strains are rapidly selected in the field. For instance, mutations in the $\beta$-tubulin gene are well known in a variety of fungi to be responsible for high levels of benzimidazole resistance $(13,18$, 21). Field strains with fenhexamid resistance (called HydR3) have been shown to carry mutations in erg27 encoding a 3-ketoreductase involved in ergosterol biosynthesis (5). Resistance to boscalid is correlated with alterations in subunits of the mitochondrial succinate dehydrogenase (15). Other mechanisms of

Corresponding author: M. Hahn; E-mail address: hahn@rhrk.uni-kl.de

* The $\boldsymbol{e}$-Xtra logo stands for "electronic extra" and indicates that the online version contains one supplemental figure and two supplemental tables.

doi:10.1094/PHYTO-02-11-0046

This article is in the public domain and not copyrightable. It may be freely reprinted with customary crediting of the source. The American Phytopathological Society, 2011 fungicide resistance are generally of lower practical relevance, such as target overexpression in Penicillium digitatum (7) or increased degradation of fungicides, which has been proposed for some $B$. cinerea genotypes with low levels of fenhexamid resistance (14). In order to reduce the risk of fungicide resistance development due to target site mutations, spraying regimes in European vineyards have been recommended that avoid the repeated use of fungicides with the same modes of action in one season.

Another drug resistance mechanism, called multidrug resistance (MDR), is well known to occur in cancer cells and various human pathogens, causing serious problems with loss of efficiency of chemical treatments. MDR is usually caused by constitutive overexpression of drug efflux transporters $(22,23)$. In plant-pathogenic fungi, MDR has been described in laboratory mutants but not been known to play an important role in field or greenhouse environments for a long time (3). In $P$. digitatum, isolates of unknown origin with resistance to both sterol demethylation inhibitors (DMIs) and cycloheximid have been reported to show overexpression of the gene encoding the $\mathrm{ABC}$ transporter PMR1 (20). In the last few years, field isolates of Mycosphaerella graminicola with high levels of resistance to DMIs and reduced sensitivity to several other fungicides have been found in many wheat-growing regions of France and in the United Kingdom. In addition to containing mutations in the DMI target gene, cyp51, these isolates showed evidence for the involvement of effluxdriven MDR (16). The mutations responsible for these MDR phenotypes in $P$. digitatum and $M$. graminicola have not been analyzed.

Starting in the mid-1990s, B. cinerea vineyard strains with MDR phenotypes have been described in rising frequencies in the 
French Champagne. The MDR strains were originally identified by their simultaneously reduced sensitivities to anilinopyrimidines and other fungicides $(2,12)$. Three MDR phenotypes were distinguished. MDR1 (AniR2) strains show reduced sensitivities to fludioxonil and cyprodinil. MDR2 (AniR3) strains show reduced sensitivities to fenhexamid, cyprodinil, and iprodione. MDR3 strains, later shown to be genetic recombinants between MDR1 and MDR2 strains, show reduced sensitivities against most fungicides used against Botrytis spp. $(2,10,15)$. In recent years, MDR has been found to represent a dominant mechanism of (partial) fungicide resistance of $B$. cinerea in the Champagne and German Wine Road regions $(10,11,15)$.

All B. cinerea MDR strains show increased fungicide efflux activities and constitutive overexpression of genes encoding drug efflux transporters (10). In MDR1 and MDR3 strains, the atrB gene encoding an ABC transporter, and, in MDR2 and MDR3 strains, the $m f s M 2$ gene encoding an MFS transporter, are constitutively upregulated. Knock-out mutants of MDR1 strains lacking $\operatorname{atr} B$ and of MDR2 strains lacking $m f s M 2$ confirmed that overexpression of these transporter genes is responsible for the MDR phenotypes. In MDR1 strains, the mutations responsible for overexpression of $\operatorname{atr} B$ were found to be located in the coding region of the $m r r l$ gene, leading to permanent activation of the encoded transcription factor Mrr1. In contrast, in MDR2 strains, overexpression of $m f s M 2$ was found to be correlated with an unusual rearrangement in the $m f s M 2$ promoter. In all MDR2 and MDR3 strains from the Champagne and the German Wine Road previously analyzed, the $m f s M 2$ promoter contained a 1,326-bp insertion from a retroelement (RE)-derived sequence in conjunction with a 678-bp deletion. By using reporter gene fusions, constitutive activation of the $m f s M 2$ promoter by the rearrangement was demonstrated (10).

In the present study, we describe the discovery of MDR2 strains from the Champagne that have a different but similar REinduced rearrangement (called type B) in the $m f s M 2$ promoter. We show that both types of mutations are correlated with the same phenotype (MDR2). We also provide population genetic evidence that only one type A and one type B founder cell has given rise to the MDR2 and MDR3 strains, respectively, isolated in three French and one German wine-growing region.

\section{MATERIALS AND METHODS}

Fungal isolates. Gray mold isolates were recovered from commercial vineyards in different years in wine-growing regions of France and Germany (Supplemental Table 1). Sampling from infected grape and cultivation of isolates was done as described previously (10). Selected field strains were used for drug sensitivity tests and Northern and Southern blot hybridizations (Table 1). B. cinerea laboratory strain B05.10 ( $m f s M 2-\mathrm{WT})$ was used as a sensitive reference strain. Strain IXa14 ( $m f s M 2$-type A) is a descendant of a cross from 1994 between SAS405 ( $m f s M 2-W T$ ) and B.692 ( $m f s M 2$-type A), isolated in the Champagne in 1994 (2).

Drug sensitivity tests. Fludioxonil (Syngenta-Agro, Maintal, Germany), fenhexamid (Bayer Crop Sciences, Monheim, Germany), and iprodione (BASF, Ludwigshafen, Germany) were kindly provided by the companies. Tolnaftate and cycloheximide were purchased from Sigma-Aldrich (St. Louis). The drugs were dissolved in $100 \%$ ethanol and added to the medium (malt extract broth; pH 5.5; Difco: Becton, Dickinson and Company, Sparks, $\mathrm{MD})$ at the required concentrations. For dilution series, fungicide stock solutions were adjusted to keep final solvent concentrations at 0.2 to $1.5 \%$ ( vol/vol). Control assays revealed no significant growth inhibition of the strains in media with $\leq 1.5 \%$ ethanol (not shown). For each isolate, $2 \times 10^{5}$ conidia were preincubated for $1.5 \mathrm{~h}$ in $1 \mathrm{ml}$ of malt extract broth before use. Minimal inhibitory drug concentrations were determined with 1,000 conidia in $0.1-\mathrm{ml}$ 96-microplate cultures, using threefold drug dilution series start- ing with $0.001 \mu \mathrm{g} \mathrm{ml}^{-1}$. After $48 \mathrm{~h}$ of incubation at $20^{\circ} \mathrm{C}$ in the dark, growth was determined by microscopy. Assays were repeated three times. Tolnaftate at concentrations $>25 \mu \mathrm{g} \mathrm{ml}{ }^{-1}$ could not be tested due to limited solubility.

Nucleic acid manipulations. DNA and RNA isolation and manipulations were performed according to standard protocols. For sequencing, polymerase chain reaction (PCR) fragments were purified using the NucleoSpin Extract II kit (Macherey-Nagel). Sequencing reactions were performed by Seq-IT GmbH \& Co. KG (Kaiserslautern, Germany). Primer pair Prom_Mfs2_1fw and Prom_Mfs2_2rev was used to amplify and sequence the $m f s M 2$ promoter region of MDR2 type A and B strains. For genomic Southern hybridization, the ${ }^{32} \mathrm{P}$-labeled probe was a mixture of fragments representing Boty 3 type A (1,267 bp) and type B (977 bp) sequences, generated by PCR with $B$. cinerea genomic DNA using primers RT-MDR2rev and RT-MDR2fw (type A) and RTMDR2rev and MDR2-TypB_fw (type B). For Northern hybridization, the probe was a 1,402-bp PCR fragment of the $m f s M 2$ coding region, amplified with primers BcM2_mfs_f and BcM2_mfs_r. For the generation of mini- and microsatellite (MS) markers (MS A, MS B, MS C, and MS D) in the genomic area flanking $m f_{s} M 2$, primers were designed and confirmed for their utility of genotyping different isolates. The markers were found by search for polymorphic sequences close to $m f s M 2$, based on comparison of the published genome sequences of $B$. cinerea strains T4 and B05.10. The repeating units in the satellite DNA of MS A, B, C, and D are (TTTGAATXTATGAAATXC),$($ GAXAX GGGXGAGAAAGAA) $)_{n}$, (GGGAAT) $)_{n}$, and (CGGAGCCTTTCT) respectively. For comparison of Boty3 RE sequences, 699-bp PCR fragments were amplified using type-A-specific primers RTMDR2fwd and RT-MDR2rev_seq, and sequenced with primer RT-MDR2rev_seq (Supplemental Table 2).

Data analysis. To support significant evidence for linkage disequilibrium between MS marker distribution and MDR2 type A genotype, a $\chi^{2}$ test with calculated $P$ values was performed. For this, the quantitative distribution of alleles for each marker in type A strains and in the reference population was compared (null hypothesis $H_{0}$ : the marker size distribution is independent from the $m f s M 2$ genotype). For comparison of Boty3 sequences, the program SeaView (version 4.2.11) (6) was used to generate the dendrogram using the maximum likelihood method. Confidence in tree topology was examined by bootstrap analysis with 1,000 replicates.

\section{RESULTS}

A second rearrangement of the $m f s M 2$ promoter has occurred in French MDR2 isolates. In all previously isolated MDR2 and MDR3 strains from vineyards in the Champagne (nine strains) and the German Wine Road (eight strains), the identical rearrangement in the $m f s M 2$ promoter had occurred due to insertion of sequence probably derived from an RE (10). The RElike sequence encodes a fragment of the reverse-transcriptase

TABLE 1. Botrytis cinerea field strains analyzed in this study

\begin{tabular}{llll}
\hline Strain & $\begin{array}{c}\text { mfsM2 } \\
\text { genotype }\end{array}$ & \multicolumn{1}{c}{ Origin $^{\mathrm{a}}$} & Year \\
\hline F02.888.2-A & Type A & France (Champagne, Hautvillers) & 2002 \\
D09.3-10.2-A & Type A & Germany (GWR, Ungstein) & 2009 \\
F02.283.2-B & Type B & France (Champagne, Moulins) & 2002 \\
F05.652.2-B & Type B & France (Champagne, Hautvillers) & 2005 \\
D08.6-1.0 & Wild type & Germany (GWR, Dackenheim) & 2008 \\
D04.0-120.0 & Wild type & Germany (Freiburg) & 2004 \\
D04.0-374.0 & Wild type & Germany (Freiburg) & 2004 \\
D06.6-15.0 & Wild type & Germany (GWR, Dackenheim) & 2006 \\
F05.A655.0 & Wild type & France (Champagne, unknown) & 2005 \\
F05.A644.0 & Wild type & France (Champagne, unknown) & 2005 \\
\hline
\end{tabular}

${ }^{\text {a }}$ GWR = German Wine Road. 
$\mathrm{RNaseH}$ domains of a polymerase that is found in several fungal long-terminal repeat (LTR) REs; for example, the Boty element of B. cinerea (4). However, both LTRs are missing, and the reversetranscriptase $\mathrm{RNaseH}$ domain is incomplete. Because of its similarity to the polymerases encoded by Boty and by a similar $B$. cinerea RE called Boty-II (27), we have assigned the sequences inserted in the $m f s M 2$ promoter of MDR2 strains to a new RE called Boty3. Up to now, however, no complete copy of the predicted Boty3 RE has been identified. To test whether the Boty3induced rearrangement is universally occurring in MDR2 and MDR3 strains, more MDR2 and MDR3 isolates were analyzed for the $m f s M 2$ promoter structure; namely, 32 isolates from the Médoc, 55 from the Champagne, 1 from the Alsace, and 57 from

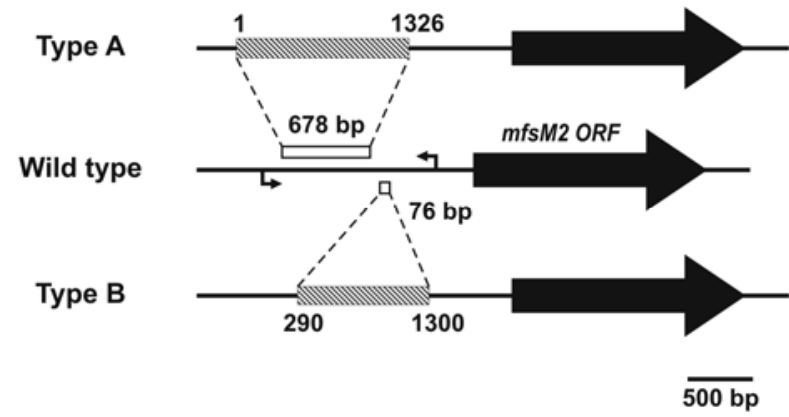

Fig. 1. Two types of rearrangements in the $m f s M 2$ promoter found in multidrug resistance (MDR)2 and MDR3 strains. Integrated retroelement-derived sequences encoding reverse transcriptase (RT) and RNaseH domains are shown as striped bars. Deleted regions are indicated as empty bars. Small arrows indicate binding sites of primers Prom_Mfs2_1fw and Prom_Mfs2_2rev. $\mathrm{ORF}=$ open reading frame. the German Wine Road wine-growing regions. Using primer pair Prom_Mfs2_1fw/Prom_Mfs2_2rev, which binds to sites flanking the Boty 3 sequence insertion in the $m f s M 2$ promoter region, these isolates were screened for size changes of the PCR product (Fig. $1)$. Whereas most of them showed the previously identified rearrangement (now called $m f s M 2$-type A), seven MDR2 isolates, recovered from different sites of the Champagne in 2002 to 2009, showed a similar but distinct rearrangement ( $m f s M 2$-type B) (Fig. 1 ), as revealed by sequence analysis. In both cases, Boty3-derived fragments of similar length and sequence $(1,326 \mathrm{bp}$ in type A, accession GQ292709; 1,011 bp in type B, accession GQ292710) have been inserted in the $m f s M 2$ promoter region but at different insertion sites. Both insertions are accompanied by deletions (type A: $678 \mathrm{bp}$; type B: $76 \mathrm{bp}$ ). Because the two deletions in the $m f s M 2$ promoter do not overlap, the idea that the two rearrangements have occurred in the same fungal cell can be excluded. The rearrangements are unusual because of the insertion of relatively small gene fragments, lacking RE-specific flanking repeats. Alignment of the type A and B RE fragments revealed that the 1,011 bp of overlapping sequences differ by 79 nucleotides (7.8\%), whereas the translated sequences differ by only $8(2.4 \%)$ amino acids (Supplemental Figure 1). The sequences of five type A RE fragments were found to be identical, and the seven type B RE fragments analyzed also had identical sequences. Although these Boty3 sequences show no similarities with the published sequences of $B$. cinerea strains B05.10 and T4, the translated sequences are $\approx 38$ and $29 \%$ (type A) and 34 and $24 \%$ (type B) identical to the homologous sequences encoded by Boty and Boty-II, respectively $(4,27)$.

The geographical origin, year of isolation, and type of RE insertion in the $m f s M 2$ promoter of all MDR2 and MDR3 isolates known so far are shown in Figure 2. It is evident that MDR2 and

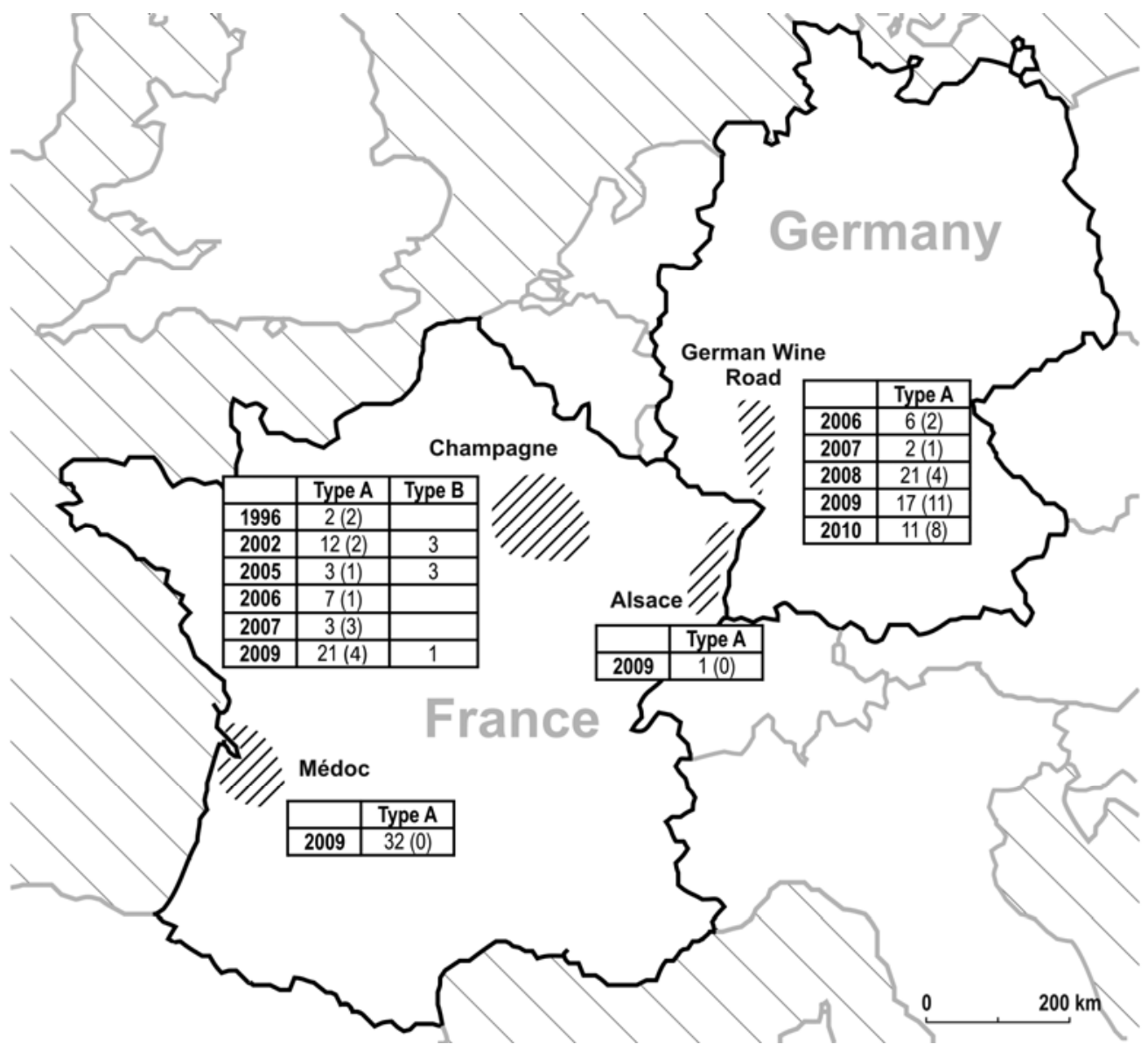

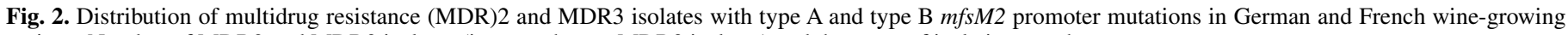
regions. Number of MDR2 and MDR3 isolates (in parentheses: MDR3 isolates) and the years of isolation are shown. 
MDR3 strains with type A insertion occur in the Médoc, Champagne, Alsace, and German Wine Road regions, whereas the type $B$ insertion was found only in MDR2 strains in the Champagne.

With $B$. cinerea reporter strains expressing $\beta$-glucuronidase under the control of $m f s M 2$ promoter fragments, we have previously shown that the $m f s M 2$ promoter in MDR2 strains is strongly activated by the type A RE-fragment insertion (10). When we compared MDR2 field isolates with either type A or type B insertions, we did not detect significant differences in their sensitivity levels toward fenhexamid, fludioxonil, iprodione, tolnaftate, and cycloheximide (Fig. 3A). In order to confirm, that both insertions lead to a similar upregulation of the $m f s M 2$ promoter, we compared the expression levels of $m f s M 2$ in MDR2 type A and type B strains. MDR2 type B strains showed very high but somewhat variable $m f s M 2$ expression levels similar to those of strains with type A insertions, whereas $m f s M 2$ expression was not detectable by Northern analysis in the sensitive control strain (Fig. 3B).

Type $A$ and $B$ Boty 3 sequences are found in many $B$. cinerea field isolates. Neither type A nor type B Boty3 RE sequences were found in the published genome sequences of $B$. cinerea strains B05.10 and T4. Therefore, we searched in the genomes of several non-MDR2/MDR3 $\mathrm{B}$. cinerea isolates of diverse geographical origins and years of isolation for the presence of similar RE sequences. PCR with total genomic DNA using type-A- or type-B-specific primers, yielded products from 11 of 13 strains. The copy numbers of these sequences were estimated by genomic Southern hybridization. For this, total DNA samples of 19 (11 non-MDR strains and 8 MDR2 strains) $B$. cinerea isolates were digested with different restriction enzymes and hybridized to a mixture of type A and type B Boty3 sequences under high-strin-

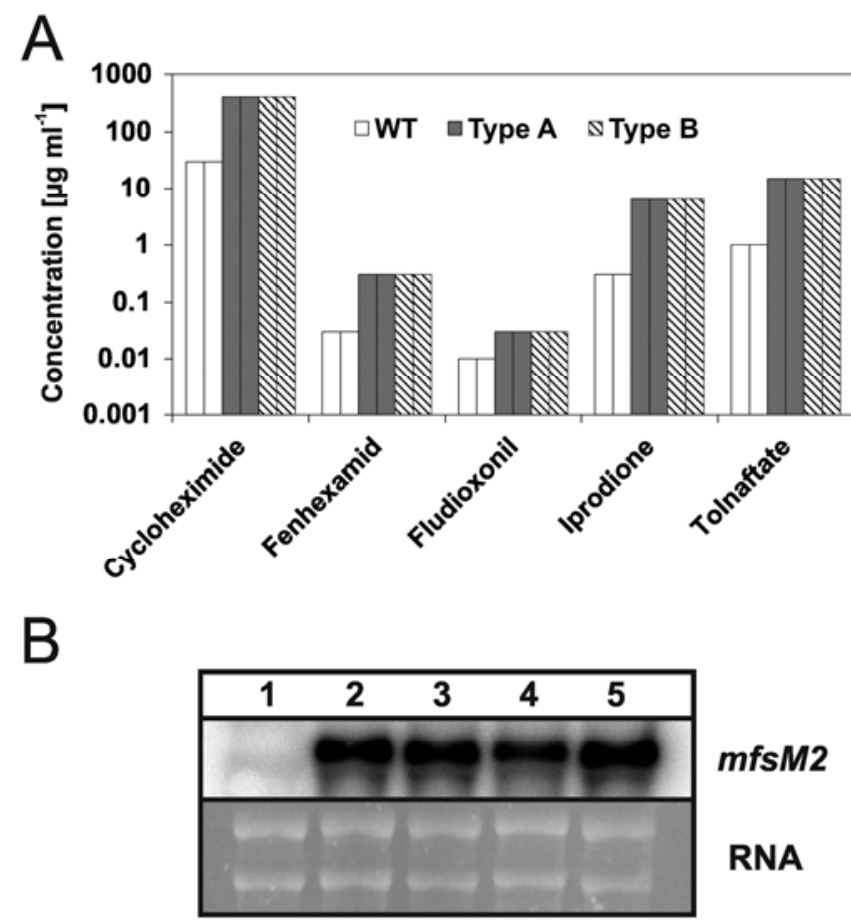

Fig. 3. Fungicide sensitivity and $m f s M 2$ expression levels of multidrug resistance (MDR) 2 isolates with type A and type B $m f s M 2$ promoter rearrangements. A, Minimal inhibitory concentrations (MIC) of the indicated metabolic inhibitors (in $\mu \mathrm{g} \mathrm{ml}^{-1}$ ). MICs are shown for two sensitive (B05.10 and D06.615.0; white bars), two type A (D09.3-10.2, F02.888.2, gray bars), and two type B (F05.A652.2, F02.283.2, striped bars) isolates. Tests were repeated three times using fungicide concentrations in threefold increments, with identical results. B, Expression levels of $m f s M 2$, analyzed by Northern hybridization. From each strain, $2 \mu \mathrm{g}$ of total RNA was loaded. Lane 1: B05.10 (sensitive); lanes 2 and 3: D09.3-10.2 and F02.888.2 (both MDR2, type A); lanes 4 and 5: F02.283.2 and F05.A652.2 (both MDR2, type B). gency conditions (Fig. 4A). The hybridization data confirmed that type A or B Boty 3 sequences are absent in the laboratory strains B05.10, T4, and SAS56 (data not shown for T4 and SAS56) and in two field isolates but present in the remaining isolates in one or a few copies. In isolates F02.888.2 and F02.283.2, the strong hybridization bands may indicate the presence of tandem repeats.

A
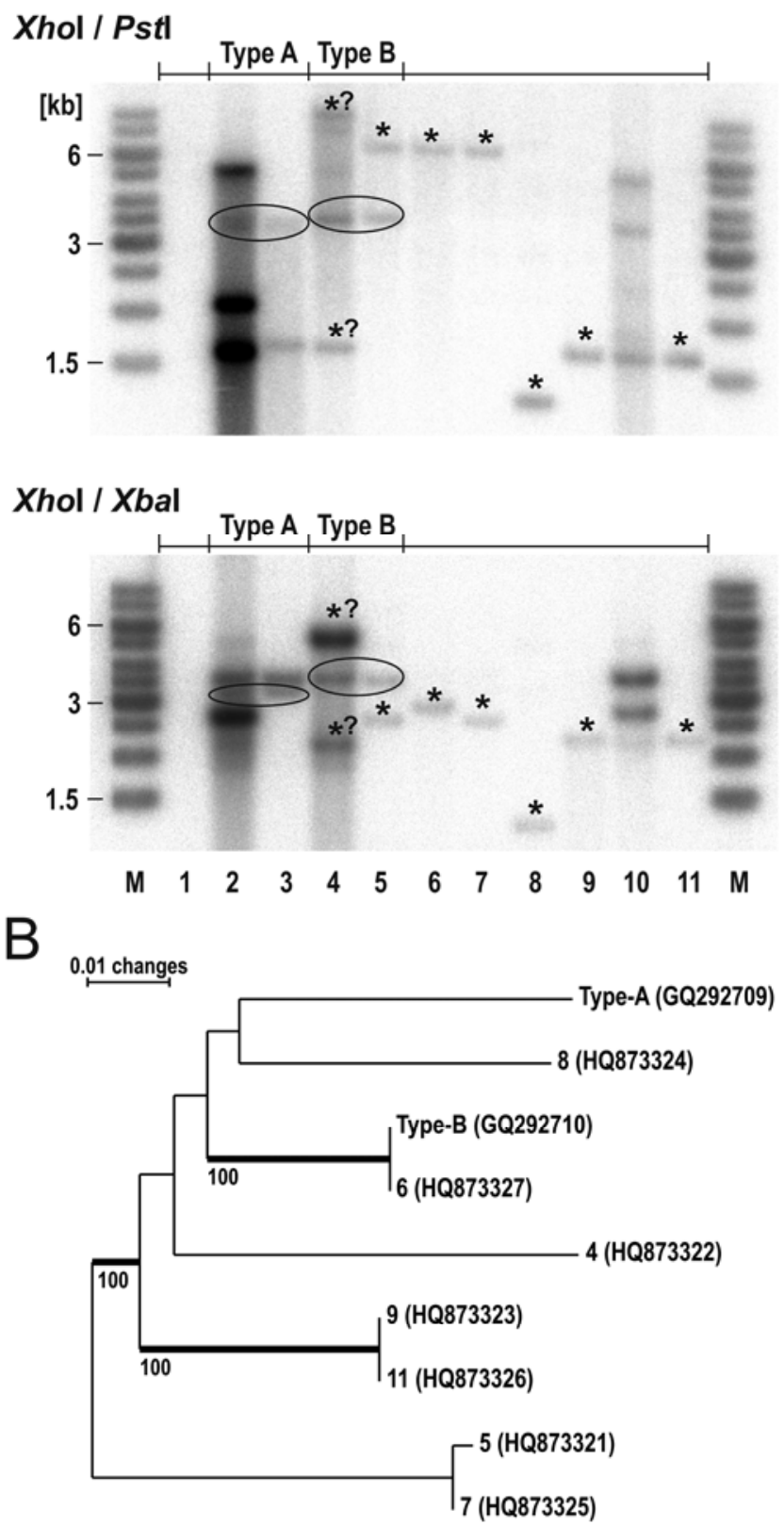

Fig. 4. Southern blot hybridization showing the presence of Boty 3 retroelement (RE) sequences in non-multidrug resistance (MDR) and MDR2 Botrytis cinerea strains. A, Southern blot hybridization using a mixture of type A and type B sequences as probe. Total DNA, digested with XhoI and PstI (upper panel) or XhoI and XbaI (lower panel), from the following isolates was loaded: lane 1: B05.10; lane 2: F02.888.2 (type A); lane 3: D09.3-10.2 (type A); lane 4: F02.283.2 (type B); lane 5: F05.A652.2 (type B); lane 6: D08.6-1.0; lane 7: D04.0-120.0; lane 8: D04.0-374.0; lane 9: D06.6-15.0; lane 10: F05.A655.0; lane 11: F05.A644.0. Fragments representing the RE insertions in $m f s M 2$ are surrounded by ovals and fragments from which sequences were probably obtained after amplification with Boty3-specific primers are marked by asterisks. For strain F02.283.2 (lane 4), it is unknown which of the bands (marked with *?) represent the sequenced DNA. B, Dendrogram showing nucleotide sequence similarities of the Boty3 RE sequences in different $B$. cinerea field strains. Numbers correspond to the lanes of the isolates analyzed in A. Branches with bootstrap values $>70 \%$ are highlighted. 
MDR2 isolates revealed more bands than those expected for the type A or type B sequences located in $m f s M 2$. The occurrence of Boty 3 bands with identical size in strains from different origins indicated that the RE sequences have conserved locations in the genomes.

To compare all Boty3 sequences, PCR was performed with genomic DNAs using primer pairs binding to either type-A- or type-B-specific sequences. Using a type-A-specific primer pair, PCR products were generated from five non-MDR strains and two MDR2 type B strains, to obtain Boty 3 sequences outside $m f s M 2$. The similarities of nine sequenced fragments are visualized in Figure 4B. The sequences are highly similar to each other, with identities of 91 to $100 \%$. The majority of sequence differences were silent, resulting in no amino acid exchanges in the reverse transcriptase coding region (not shown).

Population analysis of MDR2 and MDR3 strains. As described above, both type A and type B rearrangements in the $m f s M 2$ promoter of MDR2 and MDR3 strains are unusual, and they are identical in all MDR2 and MDR3 strains isolated in different regions and years. We have previously proposed that the type A rearrangement originally occurred in the Champagne, followed by propagation of the resulting MDR2 strains and migration from the Champagne to the German Wine Road region (10). This hypothesis is supported by the following observations: (i) MDR2 strains have been isolated first in the Champagne and (ii) the average frequency of MDR2 or MDR3 strains in the Champagne is higher than in any other wine-growing region in France (A.-S. Walker, unpublished data). For the type B rearrangement, we similarly postulate that it has occurred only once. Because all seven type B isolates found so far are from the Champagne, we propose that the type B mutation has spread within but maybe not outside of this region. To support the hypothesis of a single origin for both rearrangements, a markerbased analysis of the degree of conservation of the genomic region close to $m f s M 2$ was performed. To this purpose, polymorphic MS markers (regions containing variable numbers of 6to 18-mer repeats; see Materials and Methods) were identified in the $B$. cinerea genome, located $125,210,230$, and $1,180 \mathrm{~kb}$ downstream from $m f s M 2$. Because the supercontigs of the genome sequences from both $B$. cinerea strains terminated close to the $m f s M 2$ upstream region, no upstream markers were analyzed. If the MDR2 (and MDR3) strains indeed have a clonal origin, this region should be particularly conserved among these strains, and the degree of conservation should decrease with increasing distance to $m f s M 2$, due to outcrossing (linkage disequilibrium). In contrast, non-MDR2 strains from the same regions should show a higher degree of polymorphism in this region, regardless of the distance from $m f s M 2$. The markers were analyzed in isolates obtained from vineyards in the Medoc, Champagne, and German Wine Road regions. A graphic representation of the marker fragment size distributions is shown in Figure 5. It is evident in all regions that $m f s M 2$-linked markers are, indeed, more similar within type A MDR2 or MDR3 isolates when compared with the corresponding non-MDR2 control populations. All 101 MDR2 or MDR3 type A isolates analyzed are identical in the proximal marker A (125 kb away from $m f s M 2)$, and 95 of these strains have the identical marker B fragment size. A higher diversity is observed for marker fragments $\mathrm{C}$ and $\mathrm{D}$. A summary and statistical evaluation of the marker analysis is shown in Table 2. Compared with the non-MDR2 strains, the marker fragment size polymorphism of MDR2 or MDR3 strains is significantly different $(P<0.01)$ for all markers, except for the most distal marker D in the Médoc and Champagne populations. The seven type B MDR2 strains analyzed are identical for all markers flanking $m f s M 2$; however, because of the small number of strains, no statistics was applied here.

Comparison of the type A MDR2 or MDR3 populations of the three wine-growing regions revealed that the isolates from the
Médoc show the highest marker diversity in the $m f_{s} M 2$ region (nine haplotypes), whereas only five haplotypes can be found in Germany and four in the Champagne. One haplotype (pattern I) is dominating in all regions, representing 38\% (Médoc), 56\% (Champagne), and $49 \%$ (Germany) of the MDR2 or MDR3 isolates (Fig. 5).

\section{DISCUSSION}

In $B$. cinerea, two different types of MDR-related mutations have been identified. Strains with the MDR1 phenotype contain mutations in the mrrl gene leading to amino acid exchanges and constitutive activation of the Mrr1 transcription factor, which controls expression of atrB (10). In MDR strains of the yeasts Saccharomyces cerevisiae and Candida albicans, similar "gainof-function" mutations have been identified in two transcription factor genes, also resulting in overexpression of drug efflux transporter genes $(1,19)$. In the cases mentioned above, point mutations at different positions within the coding region of the transcription factor genes led to similar phenotypes. Therefore, these mutations are likely to occur repeatedly under appropriate selection pressure. In contrast, the mutations responsible for the MDR2 phenotype are located in the promoter region of the efflux transporter gene $m f s M 2$, and result from rearrangements induced by insertion of gene fragments derived from a predicted RE called Boty3. The type A and type B MDR2 strains were found to be indistinguishable in their fungicide resistance phenotypes and in their levels of $m f s M 2$ overexpression. Both rearrangement mutations convert the very weak $m f s M 2$ promoter into a very strong, constitutive promoter. As previously shown, the type A rearrangement resulted in an increase of $m f s M 2$ expression by a factor of $>600$ (10). The comparable $m f s M 2$ expression levels in type B MDR2 strains also suggest this promoter rearrangement to be responsible for the MDR2 phenotype. We assume that the 1,011-bp overlapping region of the type A and B inserted sequences contains a transcription factor binding element that acts as a strong activator of $m f s M 2$.

The Boty 3 sequences in the $m f s M 2$ promoters of MDR2 strains are missing in the genomes of B. cinerea strains T4 and B05.10. They were not found in the published genome sequences, nor could they be detected by PCR or genomic Southern hybridization. However, Boty3-like sequences were found in 14 of 16 field strains from diverse geographical origins, including nonMDR2-strains. As reported previously, Boty3 sequences share the highest similarity to sequences of the REAL RE of Alternaria alternata (9), and are more distantly related to Boty and Boty-II of $B$. cinerea $(4,27)$. Boty-related sequences encoding complete and truncated versions of the reverse-transcriptase RNase $\mathrm{H}$ occur in $\approx 10$ and 20 copies in the genomes of $B$. cinerea strains T4 and B05.10, respectively (not shown). Boty-II-related sequences occur in lower copy numbers (approximately nine and four copies in T4 and B05.10, respectively). Precise copy numbers cannot be determined because the annotation of these REs in the two genome sequences is still incomplete, and they are frequently found as fragments. From the results of the Southern hybridizations, we estimate the copy number of the Boty 3 sequences in most strains to be low (between zero and four), except for a few strains (e.g., F02.888.2) which show evidence for tandem insertions. The Boty3 sequences were found to be polymorphic, showing a sequence divergence of $\leq 9 \%$. This is similar to the Boty and Boty-II copies, which are also present in the $B$. cinerea genome in very similar but nonidentical copies. Which of the Boty3 sequences that are unlinked to $m f s M 2$ belong to complete REs or just fragments thereof remains to be studied. In general, the small number of Boty 3 copies, their apparently conserved locations in different strains, and the sequence polymorphism of these copies indicate a rather low transposition activity of Boty3. On the other hand, the predominantly conservative nucleotide exchanges be- 


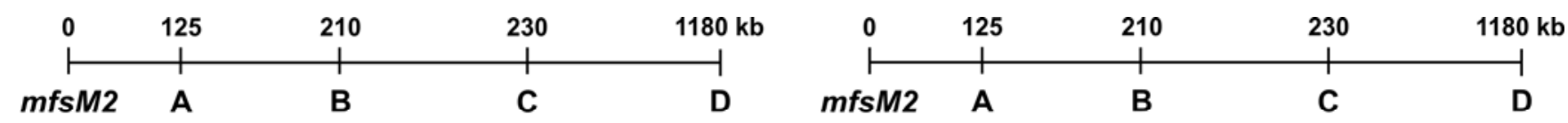

Mèdoc
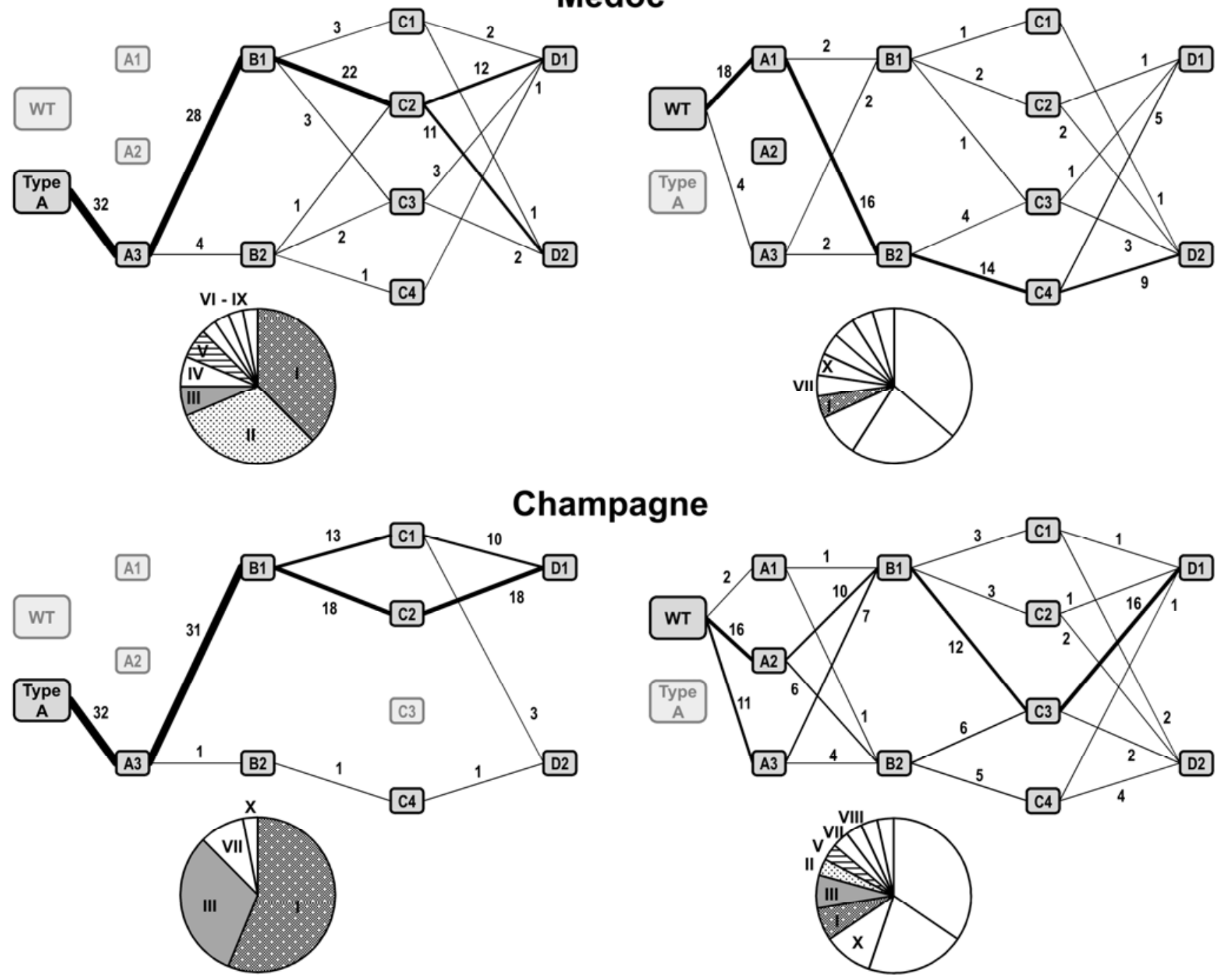

German Wine Road

C1
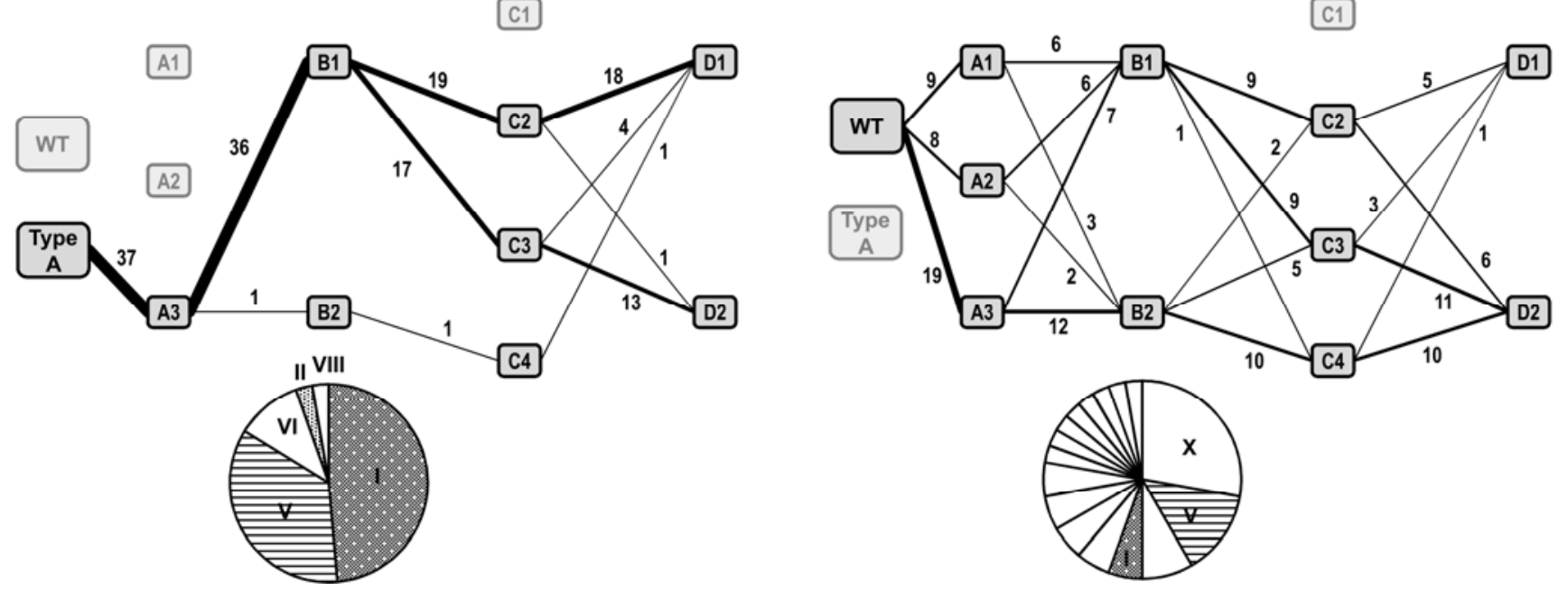

Fig. 5. Graphical representation of marker polymorphism in the genomic region flanking $m f s M 2$ in Botrytis cinerea populations from three wine-growing regions. Frequency of type A multidrug resistance (MDR)2 or MDR3 (MDR2/3) isolates (left) and non-MDR2/3 isolates (right) containing different combinations of marker sizes are illustrated. Boxes indicate different-sized marker fragments of mini- and microsatellite (MS) markers A, B, C, or D; WT = wild type. Line strength thickness and numbers next to the lines correspond to the number of isolates containing a certain marker size combination. Pie charts show the frequency of haplotypes representing different marker combinations, marked with Roman numerals and different hatching types. 
tween the copies, resulting in few amino acid exchanges, indicate that the complete Boty3 copies are active. Because of the complete absence of the LTR sequences that are typical for the gypsy family of REs, to which the Boty elements belong, it is impossible to reconstruct the formation of the two $m f s M 2$ promoter rearrangements and their possible intermediates. It is likely that, during transposition of the RE DNA into $m f s M 2$, a full-size copy of the respective (type A or type B) Boty3 element was present in the genome of the MDR2 founder cell. In subsequent generations, however, the full-size Boty3 copy might have been lost from MDR2 strains by out-crossing.

Both the type A and type B mutations in $m f s M 2$ appear to have occurred only once, as judged from the unusual nature of the promoter rearrangements, consisting of insertions of RE-derived gene fragments and small deletions at the insertion sites. Indeed, more than a hundred MDR2 and MDR3 isolates from three French and a German wine-growing region revealed an identical type A rearrangement. In order to confirm that type A and type B MDR2 or MDR3 strains are both descendants from a single founder cell, a comparative population genetic analysis of the $m f s M 2$ genomic region was performed. The analysis confirmed our hypothesis that, in MDR2 and MDR3 strains, the polymorphism of markers is zero or low in the genomic region close to $m f s M 2$, and higher with increasing distance to $m f s M 2$. In contrast, the marker variability in the $m f s M 2$ region was generally higher in non-MDR2 strains. Furthermore, the marker distribution in non-MDR2 strains was significantly different from that in MDR2 or MDR3 strains in the same regions except for the most distal marker, for which the differences were not highly significant in two cases. Remarkably, the seven type B MDR2 strains, which have been isolated in different regions of the Champagne in three different years, were found to be identical in all four markers. Collectively, these observations clearly support our hypothesis that both type A and type B strains have a single origin. Therefore, the spread of type B MDR2 strains into different regions of the Champagne and of type A MDR2 and MDR3 strains into several wine-growing regions in France and Germany is probably the result of long-distance migration events (10).

The first MDR2 isolates were recovered from a vineyard in the Champagne in 1994. Indeed, strain IXa14 (MDR2, type A) is a progeny from a cross with an MDR2 isolate from 1994 (2). Although this can be taken as an argument for the origin of MDR2 type A strains in the Champagne, the $m f s M 2$ region of MDR2 or MDR3 strains isolated in the Medoc (all from 2009) shows a higher diversity compared with strains from the Champagne and Germany. This latter observation argues for the Medoc rather than the Champagne as the site of origin for the type A mutation. More population genetic studies are necessary in order to support one of these alternatives. In particular, migration patterns and estimation of gene flow intensity between the various areas should help to decide whether the migration of MDR2 resistance from a single or limited number of origins is a correct hypothesis. At the moment, we also cannot explain the different prevalence of type A and type B populations. Our phenotypic studies do not provide evidence for differences between type A and type B regarding fungicide sensitivity of growth behavior (data not shown). Possibly, type B strains have evolved later than type A strains, resulting in a smaller and more local population.

In plant-pathogenic fungi, several cases have been reported of rare resistance mutations comparable with those leading to MDR2. All cases affect cyp51 encoding the target of sterol DMIs. In some myclobutanil-resistant strains of Venturia inaequalis, increased expression of cyp51 was correlated with the presence of a 530-bp insertion of undefined origin in the cyp51 promoter region (24). In DMI-resistant field isolates of $P$. digitatum, tandem amplification of a 126-bp sequence in the cyp51 promoter region was found to be correlated with massive cyp51 overexpression and DMI resistance (7). In the case most similar to that of MDR2 in B. cinerea, overexpression of cyp51 in Blumeriella jaapii was correlated with the promoter insertions of various truncated derivatives of a LINE-like retrotransposon in all 59 DMI-resistant isolates examined, whereas no such insertions were observed in sensitive isolates (17). Based on the insertion sites, which were not accompanied by deletions, at least two independent mutations have occurred in the B. jaapii strains. The different regional origins of strains carrying these mutations indicate that they also represent descendants from two founder cells only (17).

The appearance of MDR vineyard strains in the mid-1990s, and their increasing occurrence and spread thereafter, coincides with the introduction of novel anti-Botrytis spp. fungicides in France and Germany, in particular the anilinopyrimidines pyrimethanil and cyprodinil (since 1990) and the phenylpyrrole fludioxonil (since 1995). Because both MDR1 and MDR2 strains show increased tolerance to anilinopyrimidines, the frequent use of this fungicide class has likely contributed to their appearance in the vineyards. The frequent use of iprodione until the early 1990s might have been another factor for selection of MDR2 strains. Interestingly, the commercial use of the hydroxyanilide fenhexamid (since 2000) started several years after the first discovery of MDR2 strains in the Champagne. Nevertheless, we assume that fenhexamid has increased selection for MDR2 strains in vineyards in the last decade.

TABLE 2. Differences of multidrug resistance (MDR)2 or MDR3 (MDR2/3) and reference isolates in the region flanking $m f s M 2$, revealed by marker polymorphisms $^{\mathrm{a}}$

\begin{tabular}{|c|c|c|c|c|c|c|c|c|c|c|c|}
\hline \multirow[b]{3}{*}{ Origin, population } & \multicolumn{11}{|c|}{ Mini- and microsatellite (MS) marker } \\
\hline & \multicolumn{3}{|c|}{ MS A } & \multicolumn{2}{|c|}{ MS B } & \multicolumn{4}{|c|}{ MS C } & \multicolumn{2}{|c|}{ MS D } \\
\hline & $\begin{array}{c}\mathrm{A} 1 \\
(880 \mathrm{bp})\end{array}$ & $\begin{array}{c}\mathrm{A} 2 \\
(830 \mathrm{bp})\end{array}$ & $\begin{array}{c}\mathrm{A} 3 \\
(800 \mathrm{bp})\end{array}$ & $\begin{array}{c}\text { B1 } \\
\text { (373 bp) }\end{array}$ & $\begin{array}{c}\mathrm{B} 2 \\
(355 \mathrm{bp})\end{array}$ & $\begin{array}{c}\mathrm{C} 1 \\
(250 \mathrm{bp})\end{array}$ & $\begin{array}{c}\mathrm{C} 2 \\
(240 \mathrm{bp})\end{array}$ & $\begin{array}{c}\mathrm{C} 3 \\
(225 \mathrm{bp})\end{array}$ & $\begin{array}{c}\mathrm{C} 4 \\
(201 \mathrm{bp})\end{array}$ & $\begin{array}{c}\text { D1 } \\
(213 \text { bp) }\end{array}$ & $\begin{array}{c}\mathrm{D} 2 \\
(201 \mathrm{bp})\end{array}$ \\
\hline \multicolumn{12}{|l|}{ Médoc } \\
\hline Non-MDR2/3 & 18 & $\ldots$ & 4 & 4 & 18 & 1 & 3 & 4 & 14 & 14 & 14 \\
\hline MDR2/3 type A & 0 & $\ldots$ & 32 & 28 & 2 & 3 & 23 & 5 & 1 & 8 & 18 \\
\hline$P$ & \multicolumn{3}{|c|}{$<0.0001$} & \multicolumn{2}{|c|}{$<0.0001$} & \multicolumn{4}{|c|}{$<0.0001$} & \multicolumn{2}{|c|}{0.0196} \\
\hline MDR2/3 type A & 0 & 0 & 32 & 31 & 1 & 13 & 18 & 0 & 1 & 28 & 4 \\
\hline$P$ & & $<0.0001$ & & \multicolumn{2}{|c|}{$<0.0001$} & \multicolumn{4}{|c|}{$<0.0001$} & \multicolumn{2}{|c|}{0.056} \\
\hline MDR $2 / 3$ type B & 0 & 0 & 7 & 0 & 7 & 0 & 0 & 0 & 7 & 7 & 0 \\
\hline \multicolumn{12}{|l|}{ German Wine Road } \\
\hline Non-MDR $2 / 3$ & 10 & 8 & 18 & 19 & 17 & $\ldots$ & 11 & 14 & 11 & 9 & 27 \\
\hline MDR2/3 type $\mathrm{A}$ & 0 & 0 & 37 & 36 & 1 & $\ldots$ & 19 & 17 & 1 & 23 & 14 \\
\hline$P$ & & $<0.0001$ & & \multicolumn{2}{|c|}{$<0.0001$} & \multicolumn{4}{|c|}{0.001} & \multicolumn{2}{|c|}{$<0.0001$} \\
\hline
\end{tabular}

a Number of strains containing each marker fragment size in type A, type B, and non-MDR2 or MDR3 populations from three wine-growing regions are shown. 
In summary, MDR2 in Botrytis cinerea represents a unique example for the molecular basis and spread of a novel type of fungicide resistance in agricultural environments. A singular feature of MDR2 is that it involves the activation of a gene ( $m f s M 2$ ) that has no obvious function and is very weakly expressed under all tested conditions in non-MDR2 strains (10). Although the formation of two $m f_{s} M 2$ rearrangements are probably extremely rare events, they have been conserved by subsequent selection of the resulting MDR2 strains. Their reduced sensitivity to several fungicides - in particular, fenhexamid, cyprodinil, and iprodionehas led to their increasing occurrence in commercial vineyards and, for type A strains, to migration over long distances into new wine-growing regions. We assume that this migration toward Germany has probably occurred via translocation of conidia by air currents, which are predominantly eastward in central Europe. In France and Germany, a major part of the MDR2 populations has increasingly acquired (by sexual crosses or mutations) MDR1related mutations, leading to MDR3 phenotypes with further reduced fungicide sensitivities $(10,11)$. Because of their potential relevance for reduced effectiveness of fungicide treatments in controlling gray mold of grape, it will be of particular interest to follow the further spread of MDR2 and MDR3 populations.

\section{ACKNOWLEDGMENTS}

This work was supported by the German Science Foundation (DFG grant HA1486/8).

\section{LITERATURE CITED}

1. Carvajal, E., van den Hazel, H. B., Cybularz-Kolaczkowska, A., Balzi, E., and Goffeau, A. 1997. Molecular and phenotypic characterization of yeast PDR1 mutants that show hyperactive transcription of various $\mathrm{ABC}$ multidrug transporter genes. Mol. Gen. Genet. 256:406-415.

2. Chapeland, F., Fritz, R., Lanen, C., Gredt, M., and Leroux, P. 1999. Inheritance and mechanisms of resistance to anilinopyrimidine fungicides in Botrytis cinerea (Botryotinia fuckeliana). Pestic. Biochem. Physiol. 64:85-100.

3. De Waard, M. A., Andrade, A. C., Hayashi, K., Schoonbeek, H. J., Stergiopoulos, I., and Zwiers, L. H. 2006. Impact of fungal drug transporters on fungicide sensitivity, multidrug resistance and virulence. Pest Manage. Sci. 62:195-207.

4. Diolez, A., Marches, F., Fortini, D., and Brygoo, Y. 1995. Boty, A LongTerminal-Repeat Retroelement in the Phytopathogenic Fungus Botrytis Cinerea. Appl. Environ. Microbiol. 61:103-108.

5. Fillinger, S., Leroux, P., Auclair, C., Barreau, C., Al Hajj, C., and Debieu, D. 2008. Genetic analysis of fenhexamid-resistant field isolates of the phytopathogenic fungus Botrytis cinerea. Antimicrob. Agents Chemother. 52:3933-3940.

6. Gouy, M., Guindon, S., and Gascuel, O. 2010. SeaView version 4: A multiplatform graphical user interface for sequence alignment and phylogenetic tree building. Mol. Biol. Evol. 27:221-224.

7. Hamamoto, H., Hasegawa, K., Nakaune, R., Lee, Y. J., Makizumi, Y., Akutsu, K., and Hibi, T. 2000. Tandem repeat of a transcriptional enhancer upstream of the sterol 14 alpha-demethylase gene (CYP51) in Penicillium digitatum. Appl. Environ. Microbiol. 66:3421-3426.

8. Hollomon, D. W., and Brent, K. J. 2009. Combating plant diseases--the Darwin connection. Pest Manage. Sci. 65:1156-1163.
9. Kaneko, I., Tanaka, A., and Tsuge, T. 2000. REAL, an LTR retrotransposon from the plant pathogenic fungus Alternaria alternata. Mol. Gen. Genet. 263:625-634.

10. Kretschmer, M., Leroch, M., Mosbach, A., Walker, A. S., Fillinger, S., Mernke, D., Schoonbeek, H. J., Pradier, J. M., Leroux, P., De Waard, M. A., and Hahn, M. 2009. Fungicide-driven evolution and molecular basis of multidrug resistance in field populations of the grey mould fungus Botrytis cinerea. Plos Pathog. 5:e1000696.

11. Leroch, M., Kretschmer, M., and Hahn, M. 2010. Fungicide resistance phenotypes of Botrytis cinerea isolates from commercial vineyards in south west Germany. J. Phytopathol. 159:63-65.

12. Leroux, P., Chapeland, F., Desbrosses, D., and Gredt, M. 1999. Patterns of cross-resistance to fungicides in Botryotinia fuckeliana (Botrytis cinerea) isolates from French vineyards. Crop. Prot. 18:687-697.

13. Leroux, P., and Clerjeau, M. 1985. Resistance of Botrytis cinerea Pers and Plasmopara viticola (Berk and Curt) Berl and Detoni to fungicides in French vineyards. Crop. Prot. 4:137-160.

14. Leroux, P., Fritz, R., Debieu, D., Albertini, C., Lanen, C., Bach, J., Gredt, M., and Chapeland, F. 2002. Mechanisms of resistance to fungicides in field strains of Botrytis cinerea. Pest Manage. Sci. 58:876-888.

15. Leroux, P., Gredt, M., Leroch, M., and Walker, A. S. 2010. Exploring mechanisms of resistance to respiratory inhibitors in field strains of Botrytis cinerea, the causal agent of gray mold. Appl. Environ. Microbiol. 76:6615-6630.

16. Leroux, P., and Walker, A. S. 2011. Multiple mechanisms account for resistance to sterol $14 \alpha$-demethylation inhibitors in field isolates of Mycosphaerella graminicola. Pest Manage. Sci. 67:44-59.

17. Ma, Z., Proffer, T. J., Jacobs, J. L., and Sundin, G. W. 2006. Overexpression of the 14alpha-demethylase target gene (CYP51) mediates fungicide resistance in Blumeriella jaapii. Appl. Environ. Microbiol. 72:2581-2585

18. Ma, Z. H., and Michailides, T. J. 2005. Advances in understanding molecular mechanisms of fungicide resistance and molecular detection of resistant genotypes in phytopathogenic fungi. Crop. Prot. 24:853-863.

19. Morschhauser, J., Barker, K. S., Liu, T. T., Blass-Warmuth, J., Homayouni, R., and Rogers, P. D. 2007. The transcription factor Mrr1p controls expression of the MDR1 efflux pump and mediates multidrug resistance in Candida albicans. Plos Pathog. 3:1603-1616.

20. Nakaune, R., Adachi, K., Nawata, O., Tomiyama, M., Akutsu, K., and Hibi, T. 1998. A novel ATP-binding cassette transporter involved in multidrug resistance in the phytopathogenic fungus Penicillium digitatum. Appl. Environ. Microbiol. 64:3983-3988.

21. Park, S. Y., Jung, O. J., Chung, Y. R., and Lee, C. W. 1997. Isolation and characterization of a benomyl-resistant form of beta-tubulin-encoding gene from the phytopathogenic fungus Botryotinia fuckeliana. Mol. Cells 7:104-109.

22. Prasad, R., and Kapoor, K. 2005. Multidrug resistance in yeast Candida. Int. Rev. Cytol. 242:215-248.

23. Rouveix, B. 2007. Clinical implications of multiple drug resistance efflux pumps of pathogenic bacteria. J. Antimicrob. Chemother. 59:1208-1209.

24. Schnabel, G., and Jones, A. L. 2001. The 14 alpha-demethylase (CYP51A1) gene is overexpressed in Venturia inaequalis strains resistant to myclobutanil. Phytopathology 91:102-110.

25. Stehmann, C., and deWaard, M. A. 1996. Sensitivity of populations of Botrytis cinerea to triazoles, benomyl and vinclozolin. Eur. J. Plant Pathol. 102:171-180.

26. Williamson, B., Tudzynsk, B., Tudzynski, P., and van Kan, J. A. L. 2007. Botrytis cinerea: The cause of grey mould disease. Mol. Plant Pathol. 8:561-580.

27. Zhao, M., Zhou, J. Y., Li, Z. D., Song, W. W., Tan, Y. J., and Tan, H. 2009. Boty-II, a novel LTR retrotransposon in Botrytis cinerea B05.10 revealed by genomic sequence. Electron. J. Biotechnol. 12(3). 